\title{
Effets des métaux lourds sur les peuplements d'oligochètes de l'Ill et de ses affluents (Haut-Rhin, France)
}

\author{
A. Rossol \\ M. Lafont 1 \\ A. Exinger ${ }^{2}$
}

Mots clés : Peuplements d'oligochètes, métaux lourds, effet toxique, Alsace.

L'analyse des sédiments de quinze stations de l'Ill et de ses affluents a révélé l'existence de contaminations par les métaux lourds qui sont associées, dans la majorité des sites prospectés, à une forte charge en matières organiques. Les peuplements d'oligochètes inféodés aux sédiments fins sont dominés par des espèces résistantes à la pollution (Limnodrilus hoffmeisteri, Tubifex tubifex, Pristinella jenkinae...) ou ubiquistes (Aulodrilus pluriseta). Les fortes contaminations métalliques des sédiments sont généralement associées à des valeurs de l'indice oligochètes IOBS $\leqslant 1$ et à des effectifs $\leqslant 1000.0,1 \mathrm{~m}^{-2}$. Elles sont corrélées négativement avec le pourcentage de Tubificidae avec soies capillaires et positivement avec le pourcentage de Tubificidae sans soies capillaires. Les métaux semblent perturber la reproduction in situ des oligochètes. Les adultes de Tubificidae sont en général moins résistants que les immatures et la stratégie de reproduction par voie asexuée pourrait constituer un facteur de résistance aux contaminations métalliques.

\section{Effects of heavy metals on oligochaete communities of the River Ill and its tributaries (Haut-Rhin, France)}

Keywords : Oligochaete communities, heavy metals, toxic effect, Alsace.

The sediments of 15 sites in the River Ill and its tributaries (French Rhine Basin) are characterized by a gradient of heavy metal content, associated with organic matter. Oligochaete communities inhabiting fine substrata are characterized by the predominance of pollution-tolerant (Limnodrilus hoffmeisteri, Tubifex tubifex, Pristinella jenkinae...) or ubiquitous species (Aulodrilus pluriseta...). Generally, a high content of heavy metals is associated with low values of the oligochaete biotic index IOBS (IOBS $\leqslant 1$ ) and low abundances of worms ( $\leqslant 1000$ worms $\left..0 .1 \mathrm{~m}^{-2}\right)$. Furthermore, the percentages of Tubificidae without hair setae are positively related to heavy metal content of the sediments and the percentages of Tubificidae with hair setae are negatively related. Moreover, the sexual reproduction of oligochaetes seems to be inhibited by heavy metals : the adults of Tubificidae worms seem to be less tolerant than their immatures and the data suggest that asexual reproduction might be a strategy of tolerance to heavy metal contamination.

\section{Introduction}

Les oligochètes sont considérés, en général, comme des organismes tolérants à la pollution. Toutefois, quelques espèces seulement sont tolérantes et peuvent proliférer dans des zones polluées (Giani 1984). Ces espèces sont en réalité résistantes à l'enrichissement en matières organiques et beaucoup

1.Laboratoire Diagnose des Systèmes Aquatiques, Division Bely, CEMAGREF, 3 bis quai Chauveau, CP 220, F-69336 Lyon Cedex 09, France.

2. Laboratoire d'Hydrologie, Faculté de Pharmacie, 74 route du Rhin, F-67400 Illkirch-Graffenstaden, France.

Texte d'une communication effectuée dans le cadre du Colloque - Limnologie appliquée et application de la Limnologie Besançon, Franche-Comté, 16-19 Novembre 1992. moins à des substances toxiques comme les métaux lourds (Slooff 1983, Lafont 1989, Smith et al. 1991).

De plus, les rejets d'origine humaine se révèlent de nature complexe et les substances toxiques sont associées, dans la plupart des cas, à de fortes teneurs en matières organiques (Giani 1984). Dans ce contexte, il est délicat de mettre en évidence une incidence propre aux métaux lourds et une approche expérimentale a généralement été privilégiée (BrkovicPopovic \& Popovic 1977, Bailey \& Liu 1980, Chapman et al. 1982, Khangarot 1991, Smith et al. 1991, Reynoldson et al. 1991, Phipps et al. 1993). Cependant, toute approche expérimentale présente l'inconvénient d'une difficulté d'extrapolation des résultats 
au milieu naturel (Slooff 1983, Gilbertson 1984, Chapman 1986, 1991).

Dans ce travail, nous avons tenté de mettre en évidence un effet spécifique des métaux lourds sur les peuplements d'oligochètes récoltés dans le milieu récepteur. Le bassin de l'Ill a été retenu, dans la mesure où la contamination métallique constitue un problème préoccupant (Lafont \& Rosso 1992).

En outre, différents travaux expérimentaux ont montré que la nature du substrat influait sur la toxicité des substances xénobiotiques (Chapman et al. 1982 : McMurtry, 1984). Nous avons, par conséquent, considéré les seuls sédiments fins dans le but de disposer de données homogènes faisant abstraction autant que possible d'un effet lié à la nature du substrat.

\section{Sites prospectés}

Les stations étudiées se situent dans le bassin versant de l'Ill (Alsace). Cette rivière prend sa source au Glassberg et vient se jeter dans le Rhin à proximité de Strasbourg, après un parcours de $217 \mathrm{~km}$.

Quinze sites ont été prospectés sur l'Ill et ses affluents, dont la Largue, la Doller, la Thur, 'la Lauch, la Fecht, la Liepvrette (affluent du Giessen), le Steinbaechlein (dérivation de la Doller); la VieilleThur (bras liant la Thur à la Lauch) (Fig. 1).

Chaque station a fait l'objet de trois relevés physico-chimiques et biologiques (juin, août et octobre 1991). Seules les stations $6,7 \mathrm{~b}$ et 14 ont donné lieu à deux relevés : l'amont de la Thur (station 7b) a été choisi comme milieu de référence au vu des résultats de la première campagne montrant l'absénce de situations non polluées. D'autre part, l'Ill à Ruelisheim (station 6) se trouvait à sec au mois d'août ; le prélèvement effectué au mois de juin sur la Fecht (station 14) était constitué par $90 \%$ de sciure de bois et il n'a pas été retenu.

$\mathrm{Au}$ total, 42 relevés physico-chimiques et biologiques ont été effectués.

\section{Matériel et méthodes}

L'analyse physico-chimique et faunistique des sédiments a été réalisée à partir de relevés effectués sur les mêmes emplacements, en considérant la même épaisseur de sédiment (10 premiers centimètres).

\subsection{Analyse physico-chimique des sédiments}

Les variables physiques et chimiques retenues font l'objet du tableau 1. Afin d'apprécier la contamination des sédiments par les métaux, nous avons calculé un indice de contamination. Pour un métal donné, cet indice est défini par le rapport : Ic = concentration dans le milieu / concentration de référence admise dans le bassin Rhin-Meuse (Tableau 1). En ce qui concerne l'aluminium, le fer et le manganèse, la valeur de référence n'est pas établie au niveau du bassin; nous avons alors choisi les concentrations les plus faibles observées au niveau des 42 relevés de sédiment.

\subsection{Oligochètes}

Un carottier de type Rofès \& Savary (1981) de $25 \mathrm{~cm}^{2}$ d'ouverture est utilisé dans les sédiments fins (carottes de $10 \mathrm{~cm}$ d'épaisseur). Lorsque le sédiment se révèle trop sableux, on utilise alors un filet de type Surber de $100 \mathrm{~cm}^{2}$ d'ouverture, le substrat étant mis en suspension au moyen d'une pioche sur environ $10 \mathrm{~cm}$ d'épaisseur dans la surface délimịtée par le cadre du filet (maille de $0,160 \mathrm{~mm}$ ). Chaque échantillon est constitué dans les deux cas par trois prélèvements, soit un prélèvement tous les cinquante pas. Le matériel prélevé est stocké dans un récipient en verre et fixé sur le terrain au formaldéhyde pour obtenir approximativement une concentration de $6 \%$ de formaldéhyde dans le récipient de récupératión. Au laboratoire, les échantillons de sédiments fins sont tamisés sur un tamis de $0,160 \mathrm{~mm}$ de vide de maille. Le résidu du tamis est versé dans une cuve quadrillée de sous-échantillonnage ; 130 oligochètes sont extraits à l'aide d'une loupe binoculaire, à partir de quadrats tirés au hasard dans la cuve. Les oligochètes sont montés entre lame et lamelle dans un liquide de montage constitué par un mélange à parts égales de glycérine et d'acide lactique pur, puis déterminés à l'espèce.

\subsection{Analyses des résultats}

Pour chacun des relevés, nous avons calculé l'indice oligochètes IOBS, indice de qualité biologique des sédiments (Lafont et al. 1988). Cet indice est de la forme IOBS $=10 \mathrm{~S} . \mathrm{T}^{-1}$, où $\mathrm{S}=$ nombre total d'espèces par échantillon, $T=$ pourcentage $d u$ groupe dominant de Tubificidae (avec ou sans soies capillaires). Les plus faibles valeurs $(\leqslant 1)$ indiquent la présence d'une pollution toxique très importante, 


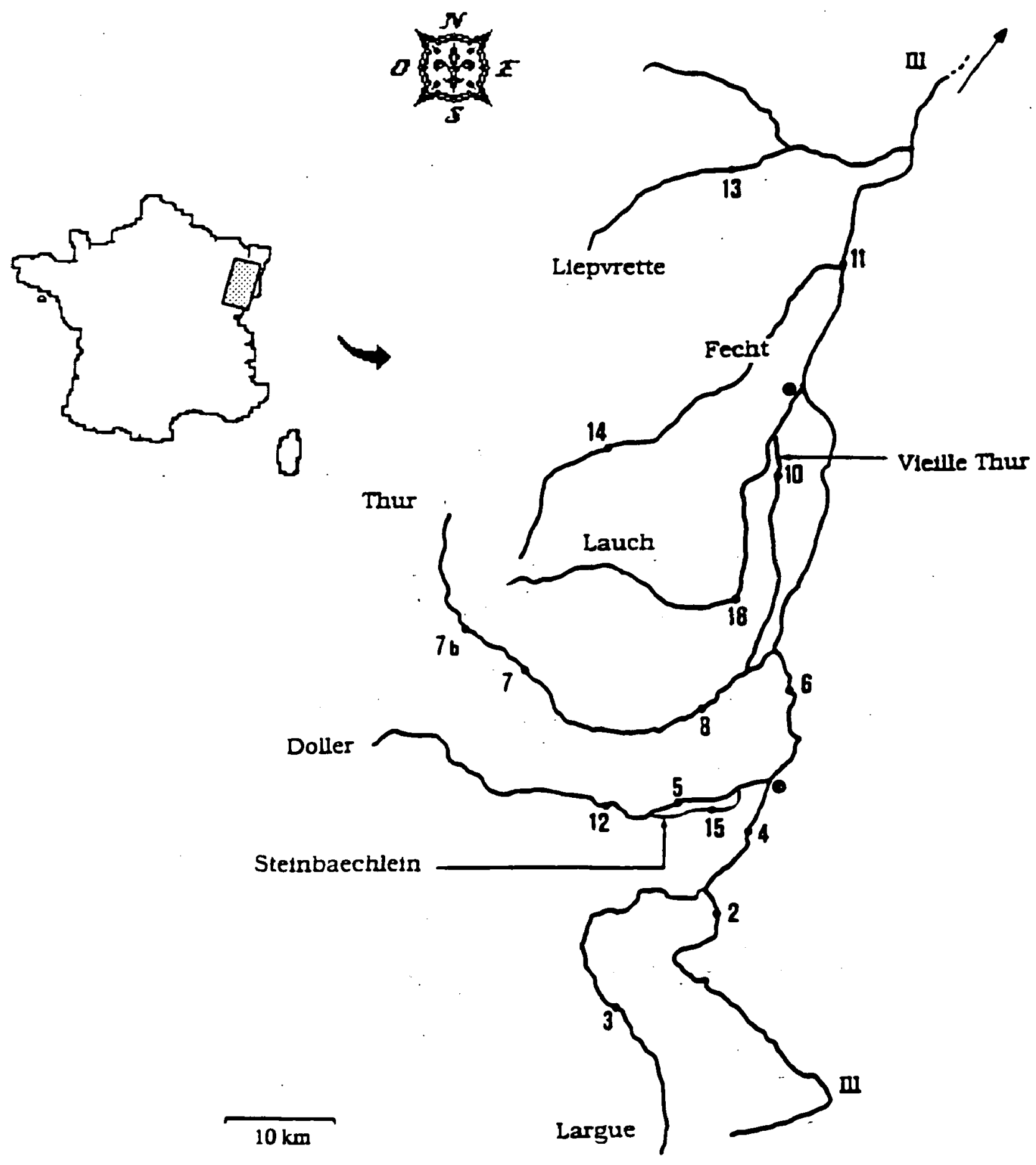

Fig. 1. Emplacement des 15 stations d'échantillonnage dans le bassin de l'Ill.

Fig. 1. Location of sampling stations in the Ill Basin.

les fortes valeurs $(>4)$ une bonne qualité biologique.

Les données biologiques et physico-chimiques ont été traitées par une analyse en composantes principales normée (ACPn) comprenant, comme variables actives, le $\log _{10}$ des variables chimiques et le pourcentage des particules sableuses (soit 16 variables pour 42 relevés). Les calculs et illustrations graphiques ont été effectués à partir du logiciel ADECO (Chessel et al. 1990).
Sur le plan F1-F2 de l'espace des individus, les quinze stations sont figurées par le centre de gravité de leurs relevés saisonniers. De même, les centres de gravité des classes de variables biologiques sont représentés graphiquement sur le premier plan de l'ACPn. Dans le cas des modalités de pourcentages (Tubificidae et Naididae), nous avons exclu la station 7 (la Thur à Willer-sur-Thur) où l'absence presque totale d'oligochètes en juin et octobre ne permettait pas le calcul d'un pourcentage. 
Tableau 1. Résultats de l'analyse physico-chimique des sédiments (valeurs moyennes) ; les stations sont classées par ordre de contamination décroissante.

Table 1. Sediment chemistry of studied sites (mean values); the sites are ranked by decreasing contamination values.

\begin{tabular}{|c|c|c|c|c|c|c|c|c|c|c|c|c|c|c|c|c|c|}
\hline & & Stations & 10 & 8 & 7 & 13 & 11 & 16 & 6 & 15 & $7 \mathbf{b}$ & 2 & 12 & 5 & 4 & 14 & 3 \\
\hline carbone organique total (COT & ng.g-1 séd. sec & & 88 & 126 & 340 & 152 & 61 & 191 & 76 & 63 & 67 & 42 & 68 & 50 & 47 & 64 & 45 \\
\hline phosphore total (Phos) & 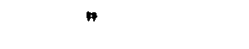 & & 2 & 2 & 2 & 2 & 2 & 2 & 2 & 1 & 1 & 1 & 1 & 1 & 1 & 1 & 1 \\
\hline azote Kjeldahl (NTK) & $n$ & & 5 & 10 & 21 & 10 & 7 & 16 & 7 & 6 & 5 & 6 & 3 & 6 & 5 & 4 & 3 \\
\hline carbonate de calcium ( $\mathrm{CaCo} 3)$ & 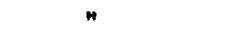 & & 2 & 3 & 3 & 3 & 4 & 5 & 11 & 8 & 1 & 31 & 3 & 3 & 30 & 1 & 12 \\
\hline Sables $\%$ séd. 0,2 à $0,5 \mathrm{~mm}$ po & $\sec$ & & 37 & 77 & 77. & 72 & 64 & 73 & 78 & 53 & 87 & 33 & 75 & 60 & .48 & 80 & 49 \\
\hline
\end{tabular}

Concentration de référence (fraction $<0,02 \mathrm{~mm}$ )

Indice de contamination $=$ concentration mesurée $($ fraction $<0,02 \mathrm{~mm})$

Al * 5920 mg.kg-1 séd. sec

$\mathrm{Mn} * 210$ "

$\mathrm{Fe}$ * 9600 "

As 7,5 "

$\mathrm{Pb} 20$ "

$\mathrm{Hg} 0,1 \quad$

$\begin{array}{lll}\mathrm{Cd} & 0,5 & \\ \mathrm{Cr} & 20 & \end{array}$

$\mathrm{Ni} 12,5 \quad$ "

$\begin{array}{lll}\mathrm{Cu} & 15 & \\ \mathrm{Zn} & 75 & \end{array}$

Micropolluants organiques

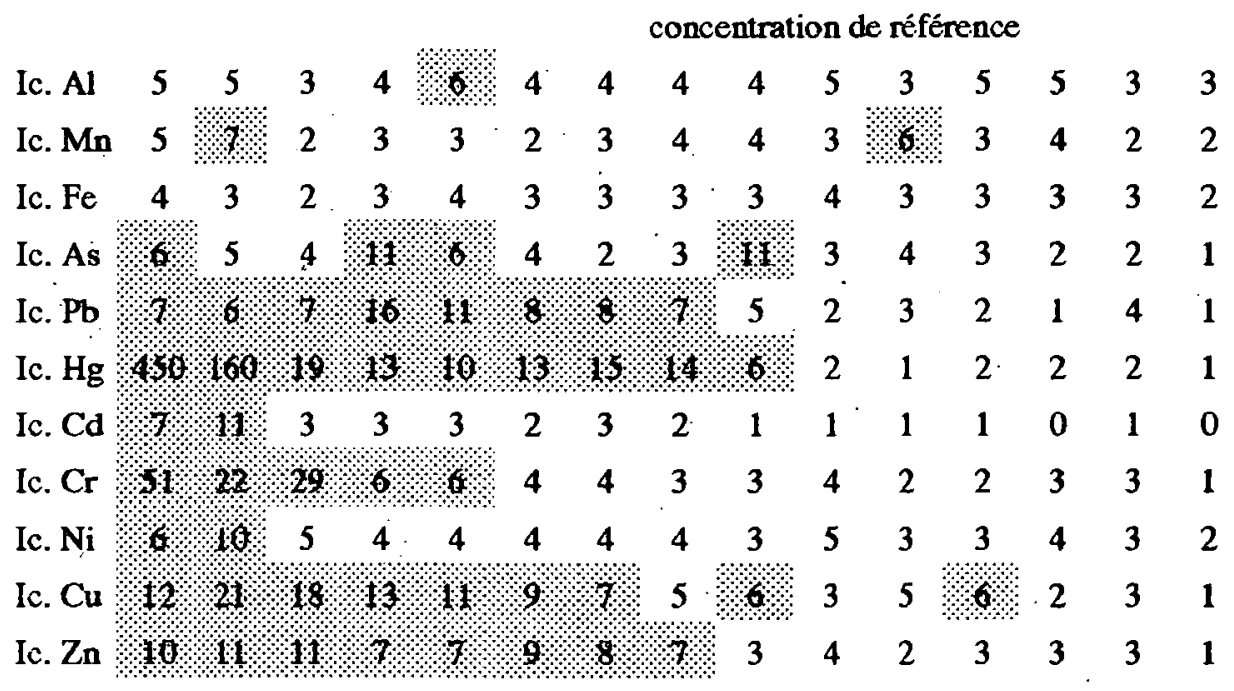

\# \# \# \#

\footnotetext{
* la concentration de référence correspond à la concentration la plus faible relevée au niveau des $\mathbf{4 2}$ analyses \# : présents

Indice de contamination $>5$
}

De plus, nous avons représenté graphiquement le $\log 10$ des effectifs des taxons sur l'axe des ordonnées en fonction de la première coordonnée factorielle de chacun des 42 relevés.

\section{Résultats}

\subsection{Physico-chimie des sédiments ; contamination des stations par les métaux lourds}

L'analyse physico-chimique des sédiments révèle une forte contamination du milieu principalement par le mercure, le zinc, le cuivre, le plomb et le chrome (Tableau 1).

Les résultats de l'ACPn permettent de mettre en évidence certaines tendances. La première composante principale $(\mathrm{F} 1=50 \%$ de l'inertie) constitue un facteur de contamination métallique des sédiments, contamination associée à la matière organique (COT, NTK, Phos) (Fig. 2a). Les variables expliquant le facteur F1 sont, dans l'ordre d'importance décroissante de leur coefficient de corrélation avec ce facteur : $\mathrm{Zn}, \mathrm{Cu}>\mathrm{Ni}>\mathrm{Cr}, \mathrm{Cd}>\mathrm{Hg}>$ $\mathrm{Pb}>$ Phos $>$ As $>$ NTK $>$ COT $>$ Fe.

La deuxième composante principale (F2 $=14 \%$ de l'inertie totale) montre surtout une opposition entre le pourcentage de particules sableuses d'une part, le fer, le manganèse, l'aluminium et le calcium d'autre part. Ce deuxième facteur oppose les sédiments sableux aux sédiments carbonatés, avec des phénomènes probables d'échanges avec les eaux souterraines, marqués par le fer et le manganèse. 
Le long du facteur F1 (Fig. 2b), les stations se répartissent selon un gradient de concentration métallique, les stations en moyenne les plus contaminées $(10,8,7,13)$ s'opposant aux moins contaminées $(3,4,2,5,12,14)$. Cependant, ces dernières stations ne sont pas exemptes de pollution, en particulier la station 3 où l'on relève en octobre des excès de matières azotées et phosphorées dans l'eau $\left(\mathrm{NH}_{4}{ }^{+}=0,32 \mathrm{mg} . \mathrm{l}^{-1}, \mathrm{PO}_{4}{ }^{3-}=1,02 \mathrm{mg} . \mathrm{l}^{-1}\right.$, Agence de l'Eau Rhin-Meuse, com. pers.). De même, la station 5 est contaminée par des pesticides (Tableau 1). On notera la position de la station 7 sur le plan F1-F2. Ce secteur sableux se révèle extrêmement chargé en métaux lourds et en matières organiques (Tableau 1).

\subsection{Peuplements d'oligochètes}

\subsubsection{Remarques générales}

Dans le bassin de l'Ill, nous avons identifié 57 unités taxonomiques. Cependant, l'examen des pourcentages d'occurrence (P.O., Tableau 2) montre que quinze taxons seulement sont récoltés au moins
7 fois (P.O. $\geqslant 15 \%$ ). Ils appartiennent à un contingent de formes universellement réputées polluorésistantes : Limnodrilus hoffmeisteri, Tubifex tubifex, Pristinella jenkinae (Giani 1984) ou ubiquistes : Aulodrilus pluriseta (Lafont 1989).

De plus, parmi les espèces les plus fréquentes, trois sont considérées comme des descripteurs d'échanges actifs entre les eaux souterraines et superficielles ( $P$. jenkinae, Pristina aequiseta (foreli), Pristina longiseta, Lafont et al. 1992). Les peuplements d'oli- gochètes du bassin de l'Ill sont probablement à l'origine diversifiés et originaux, avec des continuités d'échanges entre les milieux souterrains et superficiels. Ces peuplements se révèlent actuellement vestigiaux et dominés par quelques espèces.

Ce diagnostic pessimiste ést confirmé par l'examen des valeurs saisonnières de l'indice IOBS. En effet, $31 \%$ des sédiments présentent une très mauvaise qualité biologique (IOBS $\leqslant 1$ ), et $9 \%$ une bonne qualité (IOBS > 4).

En outre, la présence significative d'espèces d'oligochètes décrivant des échanges actifs entre le cours

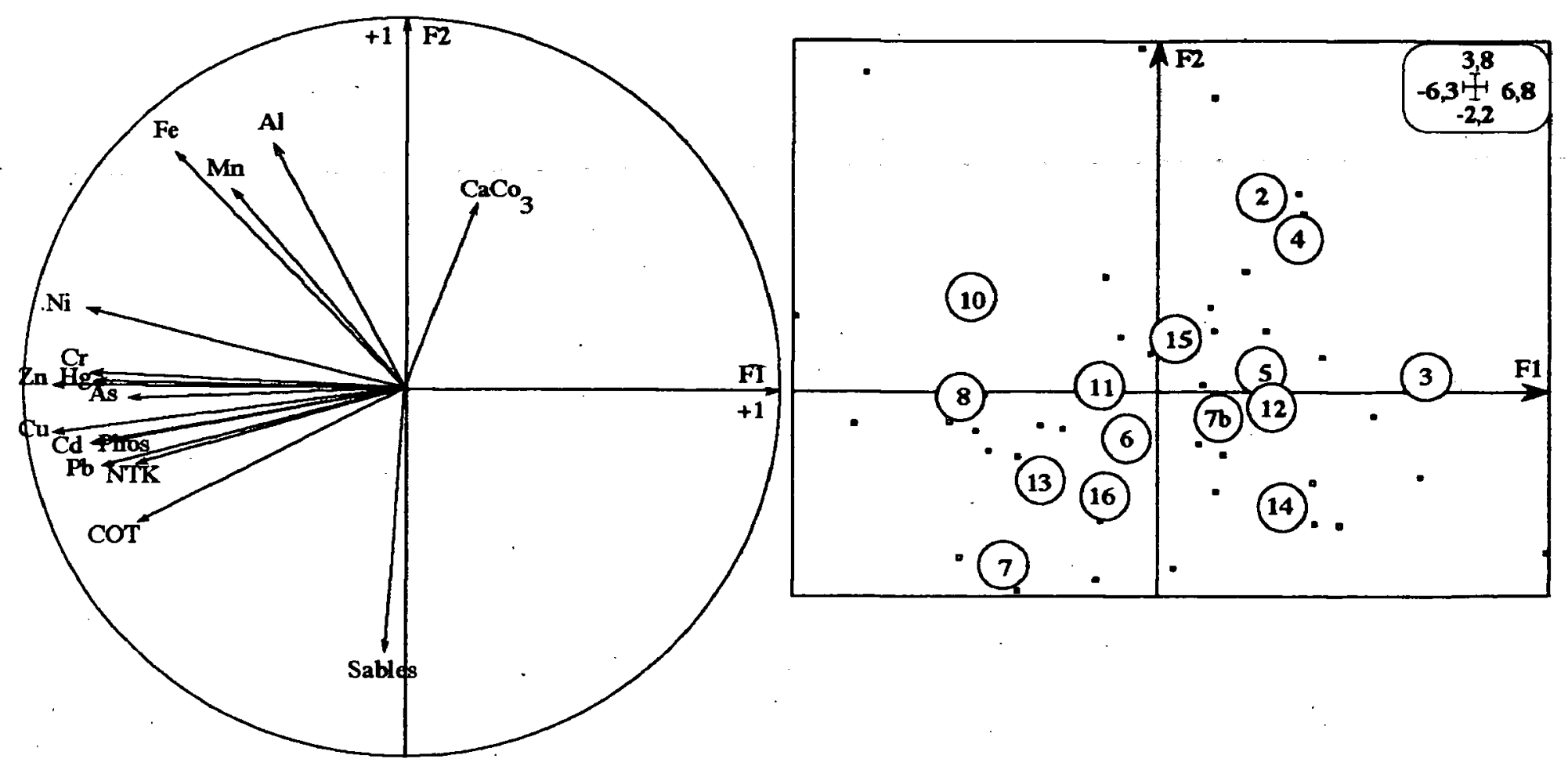

a

b

Fig. 2. Résultats de l'Analyse en Composantes Principales normée (ACPn) 2a : cercle des corrélations des 16 variables actives (facteurs F1 et F2) ;

2b : représentation des centres d'inertie des stations d'échantillonnage sur le plan F1-F2.

Fig. 2. Results of the standardized Principal Components Analysis (PCA);

2a : correlation circle of the 16 variables (factors F1-F2);

$2 b$ : plots of the inertia centers of sampling stations in the first plane of the PCA. 
Tableau 2. Liste des 30 taxons les plus fréquents (pourcentage d'occurrence P.O. $\geqslant 10 \%$ ).

Table 2. List of the 30 most frequent taxa (frequency percentages P.O. $\geqslant 10 \%$ ).

\begin{tabular}{|c|c|c|c|c|c|}
\hline TUBC & Tubificidae immatures avec soies capillaires & $\begin{array}{l}\text { P.O. } \\
93 \%\end{array}$ & TBIG & Tubifex ignotus (Stolc, 1886) & $\begin{array}{l}\text { P.O. } \\
14 \%\end{array}$ \\
\hline TUSS & Tubificidae immatures sans soies capillaires & 93 & РОНА & Potamothrix hammoniensis (Michaelsen, 1901) & 14 \\
\hline LHO & Limnodrilus hoffmeisteri Claparède, 1862 & 57 & SLAP & Slavina appendiculata d'Udekem, 1855 & 14 \\
\hline CHDS & Chaetogaster diastrophus (Gruithuisen, 1828) & 52 & VEIN & Vejdovskyella intermedia (Bretscher, 1896) & 14 \\
\hline NACO & Nais communis Piguet, 1906 & 50 & LCL & Limnodrilus claparedeanus Ratzel, 1868 & 12 \\
\hline AUPL & Aulodrilus pluriseta (Piguet, 1906) & 40 & NAPA & Nais pardalis Piguet, 1906 & 12 \\
\hline PRJE & Pristinella jenkinae * (Stephenson, 1931) & 33 & LUD & Limniodrilus udekemianus Claparède, 1862 & 10 \\
\hline ENCH & Enchytraeidae g. sp. & 33 & CADI & Chaetogaster diaphanus (Gruithuisen, 1828) & 10 \\
\hline NABA & Nais barbata Mūller, 1773 & 29 & CHII & Chaetogaster limnaei Von Baer, 1827 & 10 \\
\hline PRFO & Pristina aequiseta (foreli) * (Bourne, 1891) & 29 & AMLE & Amphichaeta leydigii Tauber, 1879 & 10 \\
\hline DEDI & Dero digitata (Müller, 1773) & 26 & NAEL & Nais elinguis Mũller, 1773 & 10 \\
\hline TBTU & Tubifex tubifex (Mũller, 1774) & 21 & VECO & Vejdovskyella comata (Vejdovsky, 1883) & 10 \\
\hline PRLO & Pristina longiseta * Ehrenberg, 1828 & 21 & DENI & Dero nivea A iyer, 1929 & 10 \\
\hline BOOO & Bothrioneurum sp. immatures & 19 & PROS & Pristinella osborni * (Walton, 1906) & 10 \\
\hline SCJO & Specaria josinae (Vejdovsky, 1883) & 17 & MAAR & Marionina argentea * (Michaelsen, 1889) & 10 \\
\hline * Esi & êces décrivant une activation des échanges & hydriques & entre & les eaux superficielles et souterraines. & \\
\hline
\end{tabular}

d'eau et la nappe est une observation inquiétante en terme de risque de contamination des eaux souterraines, problème important en plaine d'Alsace (Carbiener \& Tremolières 1990).

\subsubsection{Incidence écologique des métaux lourds du sédiment}

\section{- Incidence sur la structure des peuplements}

L'analyse graphique (Fig. 3) montre que des variables telles que le pourcentage des Naididae, les effectifs d'oligochètes ou l'indice IOBS ne sont pas reliées à la contamination métallique des sédiments. Cependant, les modalités extrêmes de l'indice IOBS ( $\leqslant$ 1 ) et des effectifs ( $\left.\leqslant 1000.0,1 \mathrm{~m}^{-2}\right)$ sont associées aux secteurs les plus chargés en métaux lourds.

De plus, le pourcentage des Tubificidae avec soies capillaires est opposé au facteur de contamination métallique, le pourcentage des Tubificidae sans soies capillaires est corrélé positivement avec ce facteur.

\section{- Incidence sur les taxons}

L'effet des métaux lourds sur l'abondance des taxons est présenté dans la Figure 4. Dans le cas des espèces ou taxons les plus fréquents et considérés a priori comme les plus résistants (pourcentage d'occurrence P.O. $\geqslant 15 \%$, Tab. 2), les immatures de Tubificidae avec soies capillaires, $T$. tubifex et Specaria josinae tendent à être significativement plus abondants ou plus fréquents dans les stations les moins contaminées. Des espèces ou taxons comme L. hoffmeisteri, N. communis, A. pluriseta, Nais barbata, $P$. aequiseta (foreli), les Enchytraeidae, Bothrioneurum sp. ou Dero digitata se révèlent indifférents aux métaux lourds.

En ce qui concerne les taxons relativement moins fréquents (P.O. compris entre 10 et $14 \%$ ), Slavina appendiculata, Vejdovskyella intermedia, Limnodrilus claparedeanus, Limnodrilus udekemianus et Amphichaeta leydigii sont opposés au facteur de contamination métallique ; 5 espèces semblent résistantes (N. pardalis, N. elinguis, $P$. osborni, $D$. nivea, $C$. diaphanus).

\section{Discussion et conclusion}

Le maintien de peuplements vestigiaux (stations 7,8 et 10 ) sur les sites les plus contaminés, mais très 
chargés en matières organiques, peut paraître surprenant. Ces observations sont probablement la résultante d'une atténuation de la toxicité des ions lourds, soit par des phénomènes de complexation. avec les matières organiques (André \& Lascombe 1987), soit par un antagonisme avec le fer et le manganèse (Roeck 1992).

Cependant, certaines variables biologiques caractérisent un effet toxique de nature métallique.

En premier lieu, les valeurs de l'indice IOBS $\leqslant$ 1 décrivent incontestablement un effet toxique global (Van Urk et al. 1985, Lafont et al. 1988), de même que les effectifs $\leqslant 1000$ individus $/ 0.1 \mathrm{~m}^{2}$. En associant ces modalités, on dispose d'un outil fiable de caractérisation de l'impact des métaux.

En second lieu, les Tubificidae sans soies capillaires prédominent dans les sédiments contaminés, observation confirmée par d'autres auteurs (Giani 1984, Chadwick et al. 1986, Ruperd et al. 1986, Burt et al. 1991, Griffiths 1991). Cette prédominance s'effectue au détriment des Tubificidae avec soies capillaires. L'absence de soies capillaires constitue un caractère morphologique qui paraît associé à une forte capacité de résistance à un facteur de toxicité (Lafont 1989). En effet, le genre Bothrioneurum (BOOO), dépourvu de soies capillaires, fait également partie des taxons résistants (Fig. 4).

En troisième lieu, on peut enrichir les listes de taxons sensibles (ou résistants) aux métaux lourds (Tableau 3). Certes, il est clair que les relations négatives métaux-espèces ne constituent que des présomptions de toxicité et non des preuves. Pour certains taxons, comme $S$. josinae ou $L$. claparedeanus, la sensibilité aux métaux lourds semble bien se

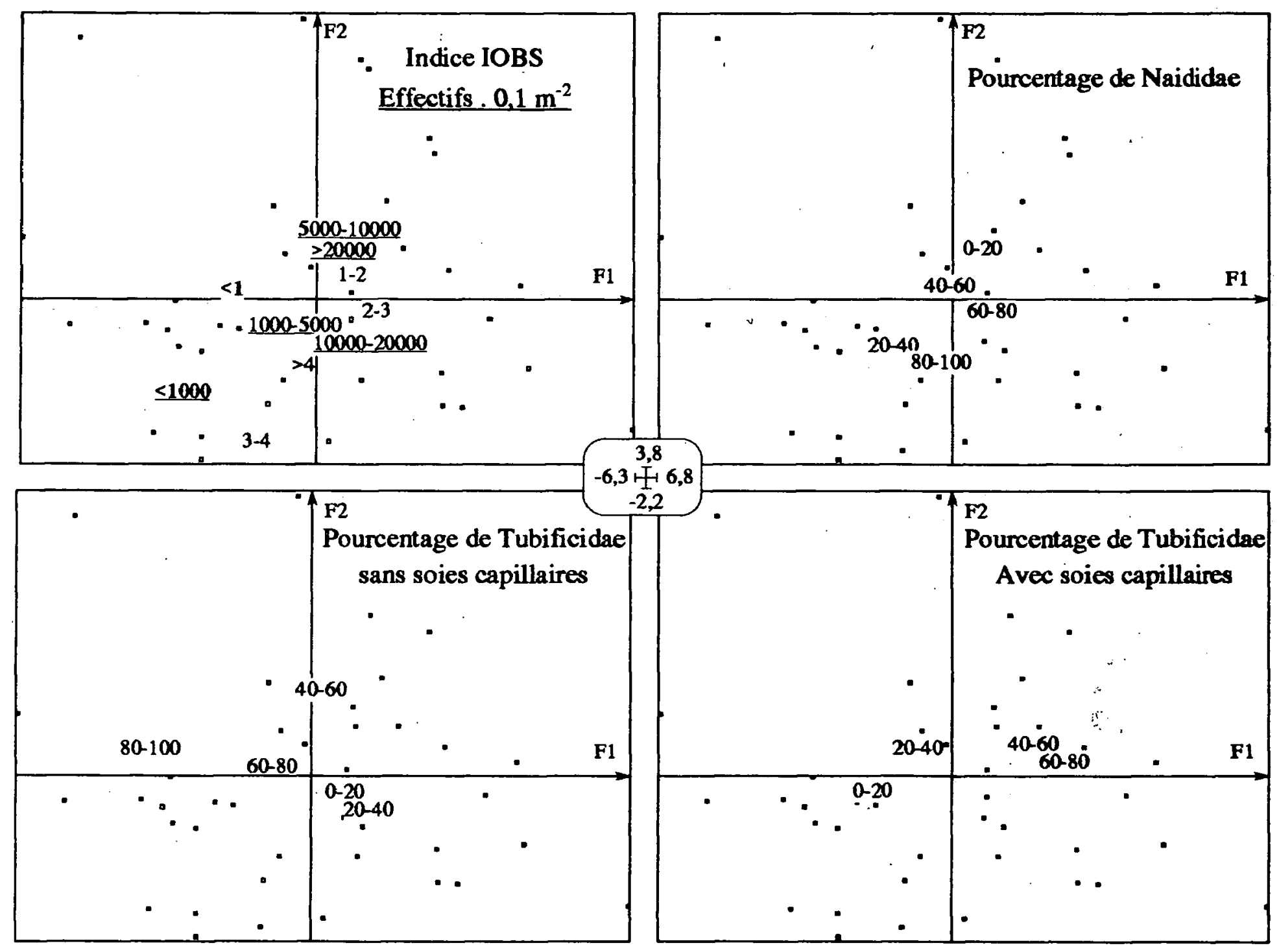

Fig. 3. Centre de gravité des modalités des variables biologiques sur le plan F1-F2 de l'ACPn.

Fig. 3. Plots of inertia centers of biological variables classes (first plane of the PCA). 

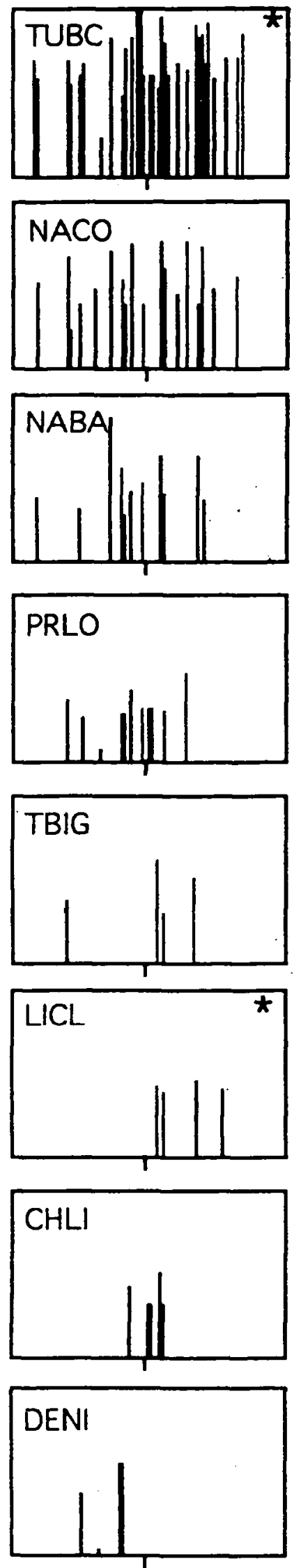
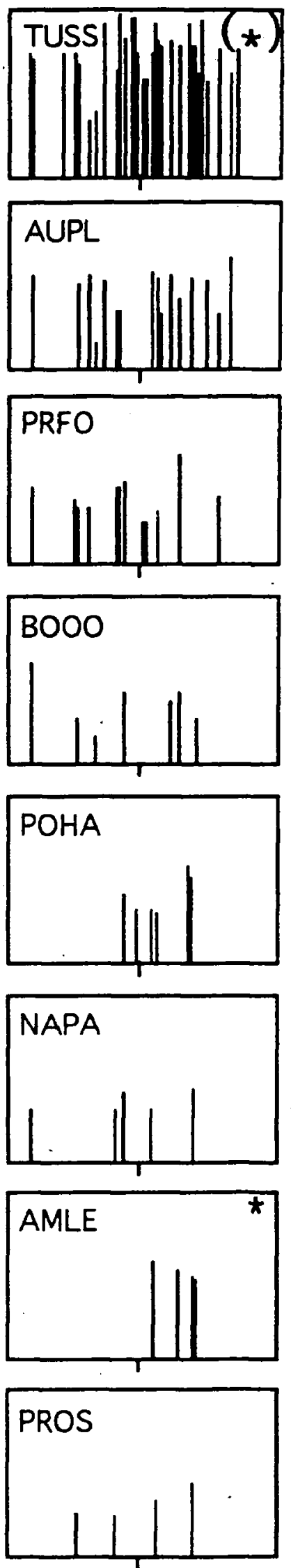
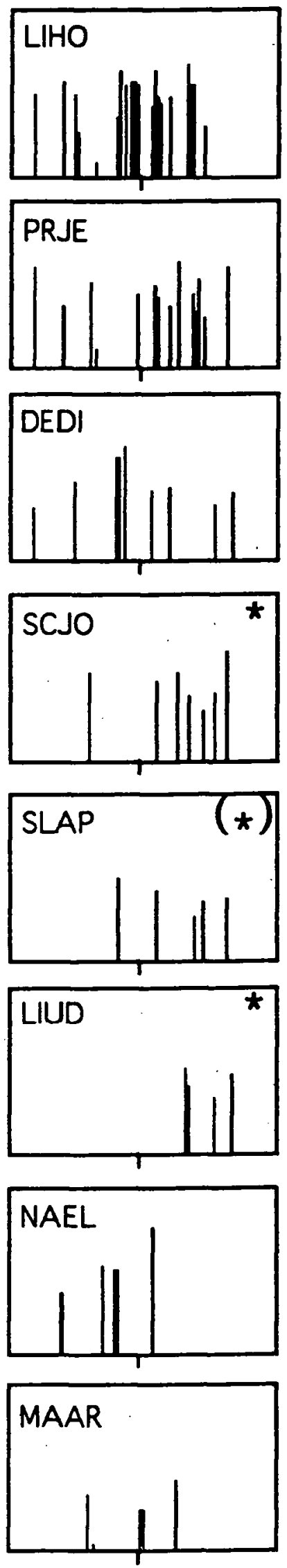
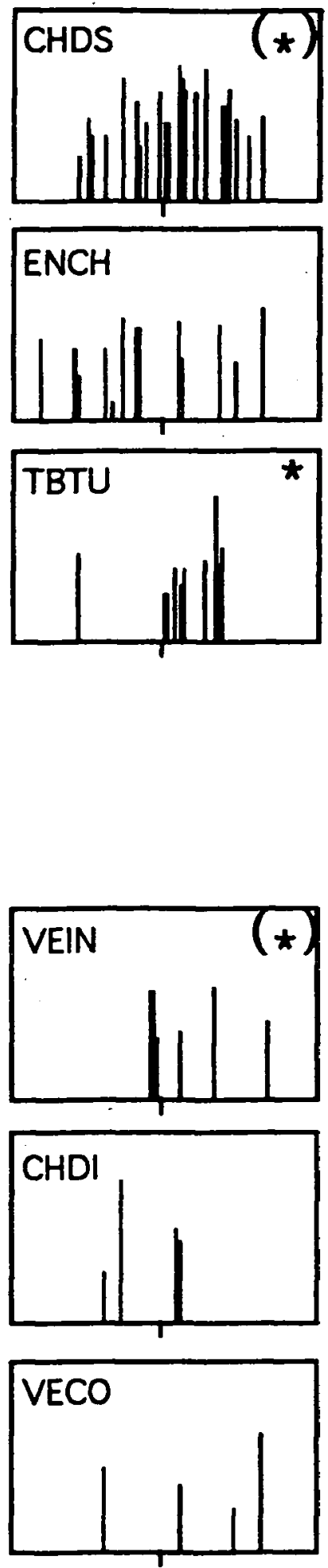

$\log _{10}$ (Effectifs+1)

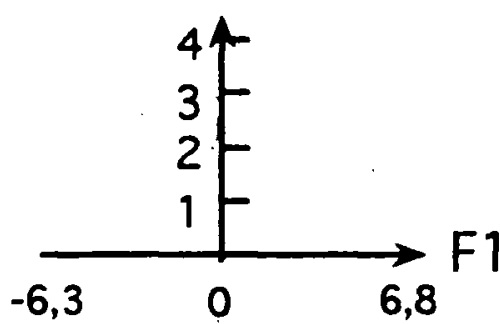

* : Taxon dont la présence et/ou l'abondance est significativement défavorisée par le facteur de contamination (test $U p<0,05$ ); $\left(^{*}\right)$ test $U p<0,1$

Fig. 4. Effectifs pour $0,1 \mathrm{~m}^{2}$ (exprimés en $\log _{10}$ ) des espèces ou taxons d'oligochètes en fonction de la valeur de la première coordonnée factorielle des $\mathbf{4 2}$ relevés. Pour la signification des codes d'identification des espèces et taxons, se reporter au Tableau 2.

Fig. 4. Abundance per $0.1 \mathrm{~m}^{2}$ (converted into $\log _{10}$ ) of oligochaete species or taxa in relation to the first co-ordinate values of the 42 surveys. For identification of species or taxa by the four letter codes, see Table 2 . 


\begin{tabular}{|c|c|c|c|}
\hline \multirow[t]{2}{*}{ Espèce ou taxon } & \multicolumn{2}{|c|}{ Métaux } & \multirow[t]{2}{*}{ Reproduction } \\
\hline & Sensible & Résistant & \\
\hline \multicolumn{4}{|l|}{ Tubificidae immatures } \\
\hline avec soies capillaires & * & & \\
\hline sans soies capillaires & * & & \\
\hline T. tubifex & * & & sexuée \\
\hline T. ignotus & * & & sexuée \\
\hline L. claparedeanus & * & & sexuée \\
\hline L. udekemianus & * & & sexuée \\
\hline P. hammoniensis & * & & sexuée \\
\hline C. diastrophus & * & & asexuée \\
\hline$\frac{S . \text { josinae }}{c}$ & * & & asexuée \\
\hline $\begin{array}{l}\text { S. appendiculata } \\
\text { C. limngei }\end{array}$ & * & & asexuée \\
\hline V. comata & * & & asexuée \\
\hline V. intermedia & * & & asexuée \\
\hline A. leydigii & * & & asexuée \\
\hline L. hoffmeisteri & & * & sexuée \\
\hline A. pluriseta & & * & asexuée \\
\hline Bothrioneurum sp. & & * & asexuée? \\
\hline C. diaphanus & & * & asexuée \\
\hline N. barbata & & * & asexuée \\
\hline N. communis & & * & asexuée \\
\hline N. elinguis & & * & asexuée \\
\hline N. pardalis & & * & asexuée \\
\hline D. digitata & & * & asexuée \\
\hline D.nivea & & * & asexuée \\
\hline P. aequiseta (foreli) & & * & asexuée \\
\hline P. jenkinae & & * & asexuée \\
\hline P. longiseta & & * & asexuée \\
\hline P. osborni & & * & asexúée \\
\hline M. argentea & & * & sexuée \\
\hline
\end{tabular}

Les taxons soulignés ont un pourcentage d'occurrence supérieur à $15 \%$.

confirmer (Lafont 1989). Inversement, le statut d'espèce sensible au cadmium, attribué à $D$. digitata (Lafont \& Rosso 1991), ne se vérifie pas ici. Le Tubificidae $A$. pluriseta ne paraît pas affecté par les métaux ; il est en effet capable de se maintenir dans des sédiments fortement contaminés (ReczynskaDutka 1985, Burt et al. 1991). En outre, des espèces considérées comme résistantes à un facteur général de pollution, telles que $P$. jenkinae (Giani 1984), $L$. hoffmeisteri, $N$. barbata, $N$. communis ou $P$. aequiseta (foreli) (Lafont 1989), présentent une forte résistance aux métaux, vraisemblablement en raison de l'affinité de ces mêmes espèces pour les matières organiques (Giani 1984, Lafont 1989). 
En dernier lieu, en considérant les quatorze taxons les plus fréquents (en excluant les Enchytraeidae qui n'ont pas fait l'objet de déterminations spécifiques), neuf espèces sont qualifiées de résistantes aux métaux (Tableau 3). Parmi ces neuf espèces, sept possèdent fondamentalement une reproduction asexuée. La stragégie de reproduction par voie asexuée favoriserait en effet la survie des espèces dans des milieux perturbés (Juget \& Lafont 1994, sous presse). De plus, l'examen de la figure 4 souligne que des adultes de Tubificidae, tels que T. tubifex et Potamothrix hammoniensis (Tubificidae avec soies capillaires) ou $L$. claparedeanus (Tubificidae sans soies capillaires), tendent à disparaître des secteurs contaminés. En revanche, les immatures correspondant à ces espèces peuvent survivre dans ces mêmes secteurs. Le déficit d'adultes de Tubificidae est à rapprocher de la possibilité qu'ont certaines espèces ( $T$. tubifex et $L$. hoffmeisteri) de changer leur stratégie de reproduction (Poddubnaya 1984, Christensen 1984) : des populations bisexuées, parthénogénétiques et asexuées peuvent effectivement coexister. Ceci constituerait une réaction de défense face à un environnement devenu défavorable (Christensen 1984). Le déficit d'adultes de Tubificidae, constaté également par Griffiths (1991), paraît donc une donnée intéressante à considérer dans les sédiments pollués par des métaux. Il semble donc se confirmer que certaines espèces d'oligochètes ont la capacité de privilégier des souches résistantes (Klerks \& Bartholomew 1991). Cette observation dans le milieu naturel peut être mise en relation avec le résultat de tests de laboratoire basés sur l'observation du cycle de développement et de reproduction des oligochètes (Reynoldson et al. 1991). Il est clair que la prise en considération, pour chaque espèce, de la relation stratégie écologique - sensibilité aux métaux lourds devrait permettre d'aboutir à des avancées théoriques et pratiques intéressantes.

\section{Remerciements}

Les auteurs remercient Mme $M$. Taillole qui a assuré la dactylographie du texte et MM. M. Babut, C. Breuzin, G. Demortier (Agence de l'Eau Rhin-Meuse), Mme M.C. Roger et MM. B. Faessel et $\mathbf{J}$. Mouthon pour leur collaboration dans ces recherches. Ces travaux ont fait l'objet d'un financement de l'Agence de l'Eau Rhin-Meuse.

\section{Travaux cités}

André B. \& Lascombe C. 1987. - Impact de la pollution métallique des cours d'eau sur les peuplements d'invertébrés benthiques : éléments de synthèse bibliographique. Document Agence de Bassin R.M.C. : 36 p.
Bailey H.C. \& Liu D.H.W. 1980. - Lumbriculus variegatus, a benthic oligochaete, as a bioassay organism. Aquatic Toxicology, ASTM STP 707. J.C. Eaton, P.R. Parrish \& A.C. Hendricks, Eds., American Society for Testing and Materials : 205-215.

Brkovic-Popovic I. \& Popovic M. 1977. - Effects of heavy metals on survival and respiration rate of tubificid worms : part I - Effects on survival. Environ. Pollut., 13 : 65-72.

Burt A.J., McKee P.M., Hart D.R. \& Kauss P.B. 1991. - Effects of pollution on benthic invertebrates communities of the St. Mareys River, 1985. Hydrobiologia, $219: 63-81$.

Carbiener R. \& Tremolières M. 1990. - The Rhine Rift Valley groundwater interactions : evolution of their susceptibility to pollution. Regulated rivers, $5: 375-389$.

Chadwick J.W., Canton S.P. \& Dent R.L. 1986. - Recovery of benthic invertebrate communities in Silver Bow Creek, Montana, following improved metal mine wastewater treatment. Water, Air and Soil Pollution, 28 : 427-438.

Chapman P.M. 1986. - Sediment quality criteria from the sediment quality triad : an example. Environ. Toxicol. Chem., $5:$ 957-964.

Chapman P.M. 1991. - Environmental criteria. What type should we be developing ? Environ. Sci. Technol., 25 (8) : 1353-1359.

Chapman P.M., Farrell M.A. \& Brinkhurst R.O. 1982. - Relative tolerances of selected aquatic oligochaetes to individual pollutants and environmental factors. Aquat. Toxicol., 2 : 47-67.

Chapman P.M. \& Mitchell D.G. 1986. - Acute tolerance tests with the oligochaetes Nais communis (Naididae) and llyodrilus frantzi (Tubificidae). Hydrobiologia, 137 : 61-64.

Chessel D., Thioulouse J., Beffy J.L. \& Auda Y. 1990. ADECO, un logiciel pour l'analyse et l'expression graphique des données écologiques (version 1.02). Document PirenVallées Fluviales, URA-CNRS 367, UCBL Lyon I.

Christensen B. 1984. - Asexual propagation and reproductive strategies in aquatic oligochaeta. Hydrobiologia, $115: 91-95$.

Giani N. 1984. - Le Riou Mort, affluent du Lot, pollué par métaux lourds. IV. Etude des Oligochètes. Annls Limnol., 20 (3) : 167-181.

Gilbertson M. 1984. - Need for development of epidemiology for chemically induced deseases in fish in Canada. Can. J. Fish. Aquat. Sci., 41 : 1534-1540.

Griffiths R.W. 1991. - Environmental quality assessment of the St Clair River as reflected by the distribution of benthic macroinvertebrates in 1985. Hydrobiologia, $219: 143-164$.

Juget J. \& Lafont M. 1994. - Theoretical habitat templets, species traits, and species richness : aquatic Oligochaetes in the Upper Rhône River and its floodplain. Freshwat. Biol. : sous presse.

Khangarot B.S. 1991. - Toxicity of metals to a freshwater tubificid worm, Tubifex tubifex (Müller). Bull. Environ. Contam. Toxicol., $46: 906-912$.

Klerks P.L. \& Bartholomew P.R. 1991. - Cadmium accumulation and detoxification in a Cd-resistant population of the oligochaete Limnodrilus hoffmeisteri. Aquat. Toxicol., 19 : 97-112.

Lafont M. 1989. - Contribution à la gestion des eaux continentales : utilisation des oligochètes comme descripteurs de l'état biologique et du degré de pollution des eaux et des sédiments. Thèse de Doctorat d'Etat ès Sciences, UCBL Lyon I : $311 \mathrm{p}$ + annexes $92 \mathrm{p}$. 
Lafont M., Coste M., Wasson J.G. \& Faessel B. 1988. - Comparaison de quatre indices biologiques pour apprécier l'impact de la pollution dans des cours d'eau français. Naturaliste Can. (Rev. Ecol. Syst.), 115 : 77-87.

Lafont M. \& Rosso A. 1991. - Impact des métaux lourds sur les peuplements d'oligochètes : résultats préliminaires. Annales de l'Institut F.A. Forel, $\mathrm{n}^{\circ}$ spécial, 3e CILEF : 26-29.

Lafont M. \& Rosso A. 1992. - Etude des peuplements d'oligochètes de quelques cours d'eau du Haut-Rhin. Description de l'impact de contaminations toxiques. Rapport CEMAGREF. Agence de l'Eau Rhin-Meuse : 49 p.

Lafont M., Durbec A. \& Ille C. 1992. - Oligochaete worms as biological describers of the interactions between surface and groundwaters : a first synthesis. Regulated Rivers, 7 : 65-73.

McMurtry M.J. 1984. - Avoidance of sublethal doses of copper and zinc by tubificid oligochaetes. J. Great Lakes Res., 10 (3) : 267-272.

Phipps G.L., Ankley G.T., Benoit T.A. \& Mattson V.R. 1993. - Use of the aquatic oligochaete Lumbriculus variegatus for assessing the toxicity and bioaccumulation of sedimentassociated contaminants. Environ. Toxicol. Chemistry, 12 : 269-279.

Poddubnaya T.L. 1984. - Parthenogenesis in Tubificidae. Hydrobiologia, 115 : 97-99.

Reczynska-Dutka M. 1985. - Ecology of some waters in the forest-agricultural basin of the River Brynica near the Upper Silesian industrial region. 4. Atmospheric heavy metal pollution of the bottom sediments of the reservoir at Kozlowa Gona. Acta Hydrobiol, Krakow, 27 (4) : 465-476.
Reynoldson T.B., Thompson S.P. \& Bamsey J.L. 1991. - A sediment bioassay using the Tubificid oligochaete worm Tubifex tubifex. Environ. Toxicol. Chem., 10 : 1061-1072.

Roeck U. 1992. - Le transfert du mercure (Hg) utilisé comme descripteur du fonctionnement hydrologique (échanges cours d'eau - nappe) dans la plaine du Rhin supérieur en Alsace. Thèse de Doctorat, Univ. L. Pasteur, Strasbourg : 195 p.

Rofès G. \& Savary M. 1981. - Description d'un nouveau modèle de carottier pour sédiments fins. Bull. Franç. Pisc., 283 : 102-113.

Ruperd Y., Lafont M. \& Cheraïtia L. 1986. - Suivi de deux retenues d'eaux pluviales de bassins versants urbanisés. Société hydrotechnique de France, XIX' journées de l'Hydraulique, Paris, 9-11 IX, 1986, Question V, Rapport 4 : 1-8.

Slooff W. 1983. - Benthic macroinvertebrates and water quality assessment : some toxicological considerations. Aquatic Toxicology, $4: 73-82$.

Smith D.P., Kennedy J.H. \& Dickson K.L. 1991. - An evaluation of a Naidid oligochaete as a toxicity test organism. Environ. Toxicol. Chem., $10:$ 1459-1465.

Van Urk G., Kerkum F.C. \& Wiersma S.M. 1985. - Bodemfauna in verontreinigde onderwater-bodems. H2O, 24 : 509-513. 